\title{
EFEITOS DA TERAPIA VIRTUAL NA FUNÇÃO MOTORA DO MEMBRO SUPERIOR PARÉTICO DE PACIENTES PÓS ACIDENTE VASCULAR ENCEFÁLICO
}

\section{EFFECTS OF VIRTUAL THERAPY ON MOTOR FUNCTION OF PARETIC UPPER LIMB OF POST STROKE PATIENTS}

\author{
Ana Carolina Lacerda Borges ${ }^{1}$ \\ Marcele Florêncio das Neves ${ }^{2}$ \\ Gabrielle Fernandes Neves ${ }^{3}$ \\ Victória Mendes de Araújo Vila Nova ${ }^{4}$ \\ Sérgio Takeshi Tatsukawa de Freitas ${ }^{5}$ \\ Eliana Aparecida Fonseca ${ }^{6}$ \\ Fernanda Pupio Silva Lima ${ }^{7}$ \\ Mário Oliveira Lima ${ }^{8}$
}

Resumo: Este artigo teve como objetivo avaliar os efeitos da Terapia Virtual (TV) no membro superior parético de indivíduos pós AVE. Participaram do estudo seis voluntários, que realizaram a terapia virtual utilizando o vídeo game XBOX 360, por meio do jogo vazamentos. Foram realizados 10 atendimentos de tratamento ininterruptos, por 30 minutos, durante duas semanas. A avaliação consistiu na quantificação da força e atividade mioelétrica no primeiro, no quinto e após o décimo atendimento. Os resultados mostraram aumento significativo da força, com consequente aumento da atividade elétrica do músculo bíceps braquial e diminuição da atividade mioelétrica para o músculo tríceps braquial $(p<0,05)$. Desse modo, acredita-se que esta forma de terapia possa ser associada aos recursos terapêuticos convencionais para acelerar o processo de reabilitação de indivíduos hemiparéticos espásticos.

Palavras-chave: Acidente Vascular Encefálico. Aprendizado Motor. Neuroplasticidade. Terapia virtual.
Abstract: This article aimed to evaluate the effects of Virtual Therapy (VT) on the paretic upper limb of post-stroke individuals. Six volunteers participated in the study, who underwent virtual therapy using the XBOX 360 video game, through the game called 'leaks'. 10 uninterrupted treatment visits were performed, for 30 minutes, for two weeks. The evaluation consisted on quantify strength and myoelectric activity in the first, sixth and after the tenth session. The results showed a significant increase in strength, with a consequent increase in the electrical activity of the biceps brachii muscle and a decrease in myoelectric activity for the triceps brachii muscle $(p<0.05)$. Thus, it is believed that this form of therapy can be associated with conventional

\footnotetext{
${ }^{1}$ Mestre, professora Centro Universitário Módulo - Cruzeiro do Sul Educacional, E-mail: carolborges.fisio@gmail.com.

$\frac{2}{2}$ Doutora em Engenharia Biomédica pela Universidade do Vale do Paraíba, E-mail: mneves@univap.br.

${ }^{3}$ Fisioterapeuta, E-mail: gabrielle.neves@hotmail.com..

${ }^{4}$ Fisioterapeuta, E-mail: victoriamaraujo@live.com.

5 Doutor, professor Universidade do Vale do Paraíba, E-mail: takeshi@univap.br

${ }^{6}$ Fisioterapeuta, E-mail: elianfonseca@yahoo.com.br.

7 Doutora, professora Universidade do Vale do Paraíba, E-mail: fpupio@univap.br.

${ }^{8}$ Doutor, professor Universidade do Vale do Paraíba, E-mail: mol@univap.br.
} 
therapeutic resources to accelerate the rehabilitation process of spastic hemiparetic patients.

Key words: Stroke. Motor Learning. Neuroplasticity. Virtual Therapy.

Data de submissão: 28.07 .2020

Data de aprovação: 10.06.2021

Identificação e disponibilidade:

(https://revista.univap.br/index.php/revistaunivap/article/view/2531, http://dx.doi.org/10.18066/revistaunivap.v27i55.2531).

\section{INTRODUÇÃO}

O acidente vascular encefálico (AVE) ocorre quando há uma isquemia ou hemorragia cerebrovascular. É a terceira causa de morte mundial e a causa mais comum de comprometimento neurológico em adultos (TANAKA et al.,2019; KIPER et al., 2018; CHOI, PAIK, 2018). Dentre as complicações do AVE há a espasticidade, definida como um distúrbio sensório motor causado por uma lesão no neurônio motor superior, gerando uma ativação involuntária intermitente ou sustentada dos músculos (TRICCAS et al., 2019). Esta pode causar diminuição da força muscular devido à diminuição, à restrição da amplitude de movimento e à redução da velocidade de movimento do membro (NAM et al., 2019).

A reabilitação neurológica tem como o principal objetivo promover a neuroplasticidade, definida como a capacidade do neurônio em alterar sua função, perfil químico ou estrutura (CARVALHO et al., 2018; LAUDY-EKMAN, 2008). A capacidade de neuroplasticidade ainda está presente mesmo no período crônico do AVE, e, além do tempo de lesão, esta depende também do tipo de intervenção, idade e local da lesão (CARVALHO et al., 2018). O treinamento específico e repetitivo associado ao estímulo cognitivo promovem sinaptogênese e melhoram a resposta motora, estimulando a reorganização cortical na reabilitação pós-AVE (IKBALI AFSAR et al., 2018; KHAN et al., 2016).

Há muitas técnicas que podem auxiliar o fisioterapeuta a alcançar os objetivos dentro da reabilitação neurológica, como a cinesioterapia, treinamento físico, hidroterapia, estimulação intracortical e terapia robótica (ARAMAKI et al., 2019). A terapia por realidade virtual é um método que vem demonstrando resultados promissores recentemente (MAGGIO et al., 2019). Através da simulação de um ambiente real, o sistema da realidade virtual permite o trabalho baseado na execução de tarefas, promovendo a intensidade de exercício necessária para gerar a neuroplasticidade (IKBALI AFSAR et al., 2018).

Considerando que a função do membro superior é afetada em grande porcentagem dos pacientes com AVE, ocasionando a dependência para a realização de tarefas e atividades da vida diária (CARVALHO et al., 2018) e considerando a terapia por realidade virtual uma promessa da reabilitação neurológica, o objetivo do presente estudo foi avaliar os efeitos da Terapia Virtual (TV) no membro superior parético de indivíduos pós AVE.

\section{MATERIAIS E MÉTODOS}

Trata-se de um estudo longitudinal, no qual foram avaliados 6 indivíduos, com 
diagnóstico médico de AVE e diagnóstico fisioterapêutico de hemiparesia espástica (53 $\pm 16,7$ anos), de ambos os sexos.

O estudo foi realizado no Laboratório de Engenharia de Reabilitação Sensório Motora da Universidade do Vale do Paraíba (UNIVAP), após aprovação pelo Comitê de Ética em Pesquisa (CEP) sob protocolo n CAAE 20749113.5.0000.5503 e Clinical Trials NCT02061371. Após os devidos esclarecimentos, os voluntários assinaram o Termo de Consentimento Livre e Esclarecido (TCLE).

Os critérios de inclusão do estudo foram: idade entre 30 e 70 anos; grau 1 a grau 2 de espasticidade nos MMSS, segundo a Escala de Ashworth Modificada; amplitude passiva total da articulação do cotovelo ao movimento de flexão e extensão; deambulação independente, com ou sem apoio; cognição preservada e com no mínimo 6 meses de lesão. Com relação aos critérios de exclusão, foram adotados os seguintes aspectos: pacientes que apresentam déficit visual e auditivo; rigidez articular e lesões osteomioarticulares do cotovelo; incapacidade de interpretação dos recursos terapêuticos a serem apresentados; afasia de Wernick e Broca; hipertensão arterial não controlada.

\subsection{PROTOCOLO DE AVALIAÇÃO E TRATAMENTO}

Todos os voluntários realizaram terapia virtual utilizando o vídeo game $X$ BOX 360, por meio do minijogo "vazamentos", por 30 minutos, 5 vezes por semana ininterruptos, durante duas semanas, totalizando 10 atendimentos. As avaliações foram realizadas antes do primeiro e do quinto atendimento, bem como ao término do tratamento, no $11^{\circ}$ dia. Foram avaliadas a força e a atividade mioelétrica do membro superior parético, por meio da dinamometria e eletromiografia de superfície.

Para mensuração da força foi utilizado um dinamômetro portátil da marca EMG System do Brasil Ltda. Os dados foram coletados com o voluntário posicionado em sedestação, mantendo os pés apoiados no chão e os ombros em abdução de $90^{\circ}$, juntamente com flexão de cotovelos e ambas as mãos segurando o equipamento. $O$ voluntário foi orientado a realizar movimento de adução das escápulas durante 20 segundos realizando força isométrica máxima.

A atividade elétrica muscular foi adquirida por um eletromiógrafo da marca EMG System do Brasil Ltda., com oito canais, composto por um conversor A/D (conversor analógico-digital) de 12 bits de resolução, calibrado com $2000 \mathrm{~Hz}$ de frequência de amostragem, unidade $\mu v$, Xmin -1000, Ymáx 1000, Coef.A 0 e Coef.B 500.

Para aquisição de sinais foram utilizados eletrodos de $\mathrm{Ag} / \mathrm{AgCl}$ (prata/cloreto de prata) aderidos à pele, constituindo uma superfície de detecção que capta corrente por meio da interface pele-eletrodo, sendo fixados à pele após higienização com algodão embebecido de álcool a $70 \%$ no local de fixação.

Os eletrodos de superfície foram colocados aos pares sobre o ponto motor dos músculos bíceps braquial e tríceps braquial, conforme o protocolo Surface-EMG for the Non Invasive Assessment of Muscle (SENIAM), acompanhando o sentido longitudinal das fibras musculares. Os dados foram coletados em sincronia com o dinamômetro, respeitando o tempo de 20 segundos.

O teste da atividade elétrica muscular foi realizado no membro superior parético e no membro superior sadio, afim de quantificar a influência dos músculos de ambos os membros durante a execução do movimento de força.

O procedimento de tratamento foi realizado com o voluntário em ortostatismo, sendo orientado a permanecer sobre uma região demarcada no chão com uma distância de 2 metros em relação ao Kinect $\circledast$, onde a imagem foi calibrada de acordo 
com a altura do indivíduo, realizada automaticamente pelo software e assim projetada na tela da televisão, desenhando um retrato em animação do usuário, oferecendo em tempo real feedback dos movimentos na tela de projeção, que ficava disposta à frente do indivíduo de forma a permitir um amplo campo de visão (Figura 1).

Ao iniciar os protocolos programados pelo jogo, o voluntário visualizava um aquário no fundo do mar e peixes que perfuravam o mesmo, e deveria então movimentar os membros superiores para sanar as perfurações em diferentes alturas e posições. Com o posicionamento realizado de forma adequada, o vazamento terminava. Ao término de uma sequência de movimentos, a pontuação atingida era mostrada na tela, bem como fotos durante a atividade, indicando o término da fase do jogo.

Neste ambiente, os voluntários jogaram as 9 fases propostas pelo jogo, dividas em básico, intermediário e avançado, durante 30 minutos, sendo instruídos a utilizar o membro superior parético durante toda terapia.

O principal intuito do jogo foi que, ao ver a si mesmo realizando exercícios e recebendo o estímulo sonoro emitido pelo software do game, ocorresse a estimulação e motivação do voluntário, fazendo com que o mesmo realizasse todos os exercícios propostos pela terapia.

Figura 1 - Terapia Virtual utilizando vídeo game X BOX 360, jogo vazamentos.

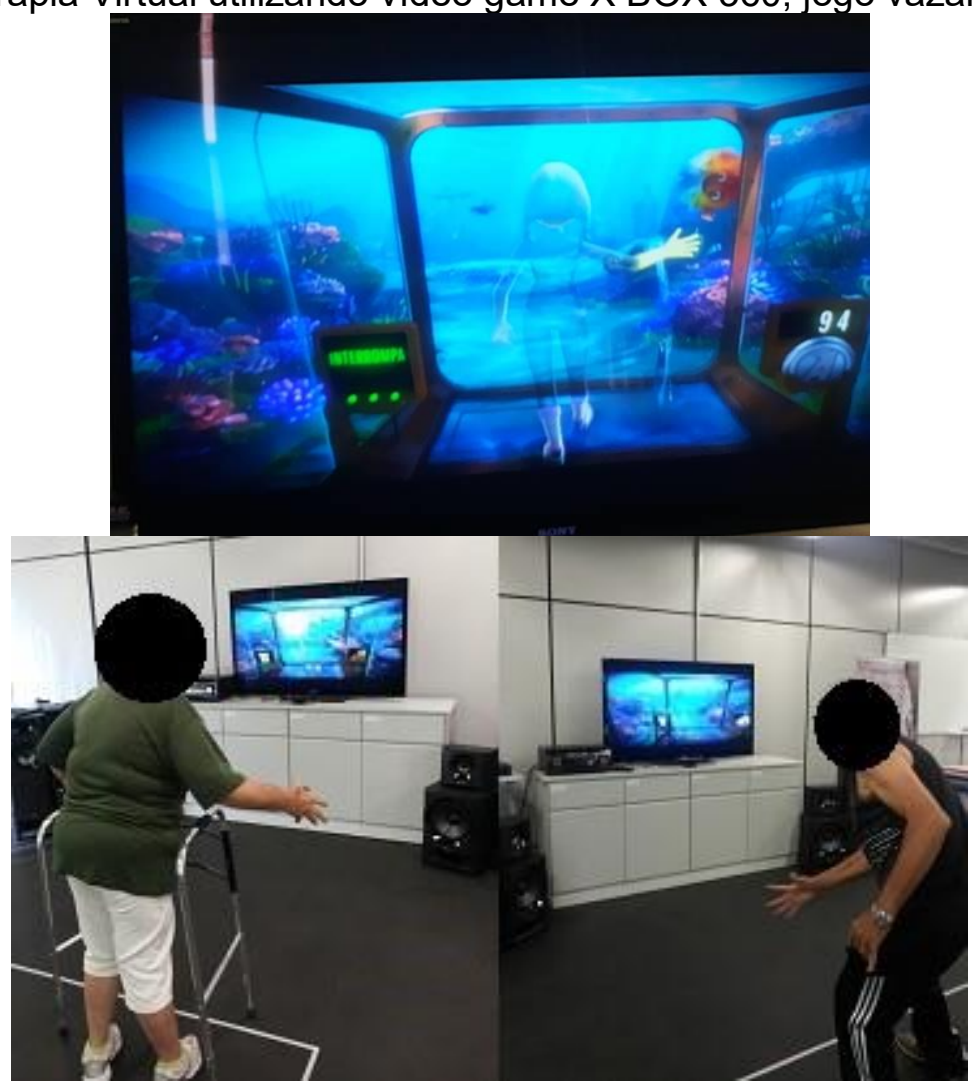

Fonte: Os autores.

\subsection{ANÁLISE DOS DADOS}

Os dados de EMG e dinamometria foram processados no software EMG Works Analysis ${ }^{\circledR}$, considerando o valor de RMS (Root Mean Square), ou seja, a raiz quadrada do valor médio quadrático e da força na contração isométrica. 
Durante a análise dos sinais, excluiu-se o primeiro segundo inicial e o último segundo final, considerando apenas os 18 segundos restantes. Com relação aos dados da eletromiografia, o sinal foi fragmentado, obtendo 3 valores médios quadráticos RMS - Root Mean Square, referente a 6 segundos de coleta, calculando então a média desses valores obtidos. Após obtenção dos valores, os mesmos foram organizados no Microsoft Office Excel.

A normalização desses dados referentes a eletromiografia e dinamometria foi realizada com relação ao valor inicial de cada paciente: dividiu-se o valor final pelo valor inicial, com o intuito de obter uma comparação pareada, garantindo a avaliação do ganho real de cada paciente.

A análise estatística foi realizada por meio do software estatístico Instat $囚$. A metodologia utilizada na análise foi descritiva e paramétrica, visto que todas as variáveis apresentaram distribuição normal de acordo com o teste de normalidade Kolmogorov-Smirnov. Para a verificação de existência de diferenças estatísticas entre as médias dos dados, utilizou-se o Teste T-Student pareado com nível de significância $5 \%(p \leq 0,05)$.

\section{RESULTADOS}

Evidenciou-se valores estatisticamente significativos com relação à força antes e após o tratamento proposto $(p=0.0058)$ (Figura 2), bem como aumento da atividade elétrica muscular do músculo bíceps braquial parético $(p=0.0323)$ (Figura 3$)$, e diminuição da atividade elétrica muscular do músculo tríceps braquial após a terapia ( $p=0.0095$ ) (Figura 4). Não foram evidenciados valores estatisticamente significativos na avaliação após a intermediária.

Figura 2 - Box Plot da média dos valores de força durante contração isométrica, realizada com auxílio do dinamômetro. $\S$ Antes VS Depois $-p=0.0058$.

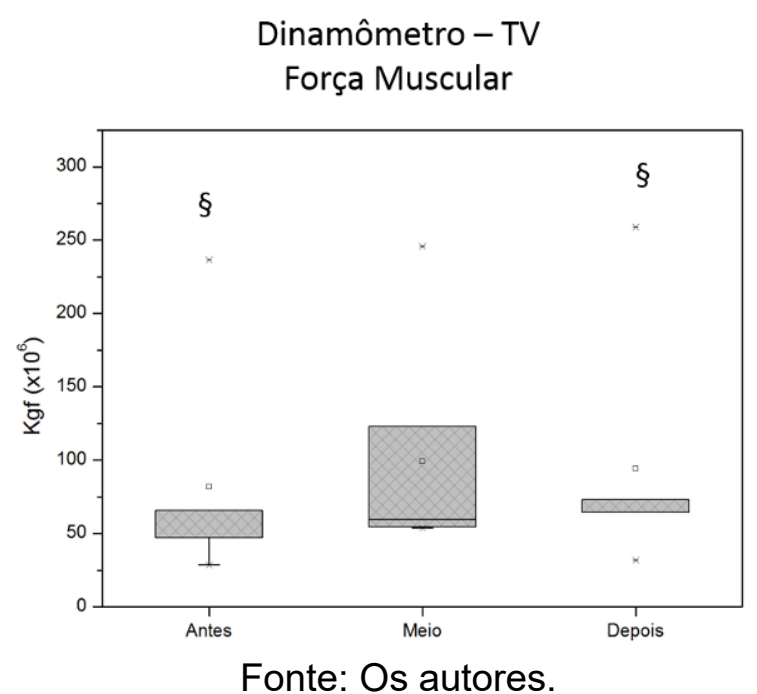


Figura 3 - Box Plot da média dos valores de RMS do sinal mioelétrico do músculo bíceps braquial espástico durante contração isométrica. § Antes VS Depois $-p=0.0323$.

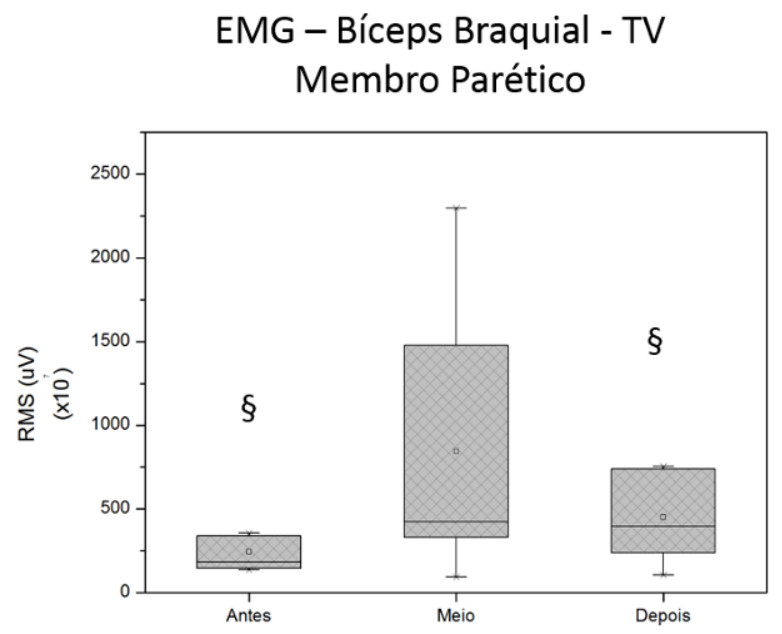

Fonte: Os autores.

Figura 4 - Box Plot da média dos valores de RMS do sinal mioelétrico do músculo tríceps braquial espástico durante contração isométrica. § Antes VS Depois $-p=0.0095$.

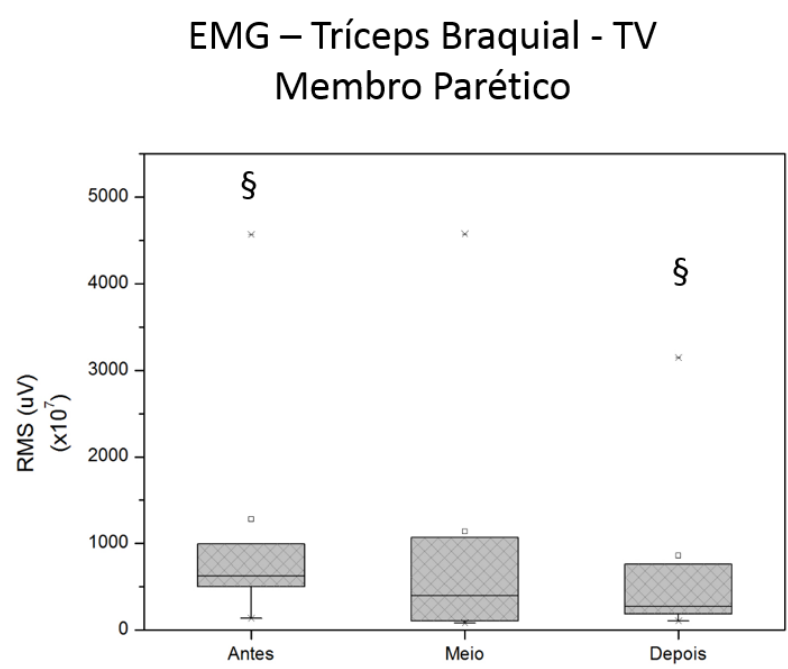

Fonte: Os autores.

Não foram constatadas mudanças significativas na atividade elétrica dos músculos estudados no membro superior sadio, demonstrando que os mesmos mantiveram o padrão do início da terapia, como pode ser observada nas figuras 5, 6 e 7. 
Figura 5 - Box Plot da média dos valores de RMS do sinal mioelétrico do músculo bíceps braquial sadio durante contração isométrica.

EMG - Bíceps Braquial - TV

Membro Sadio

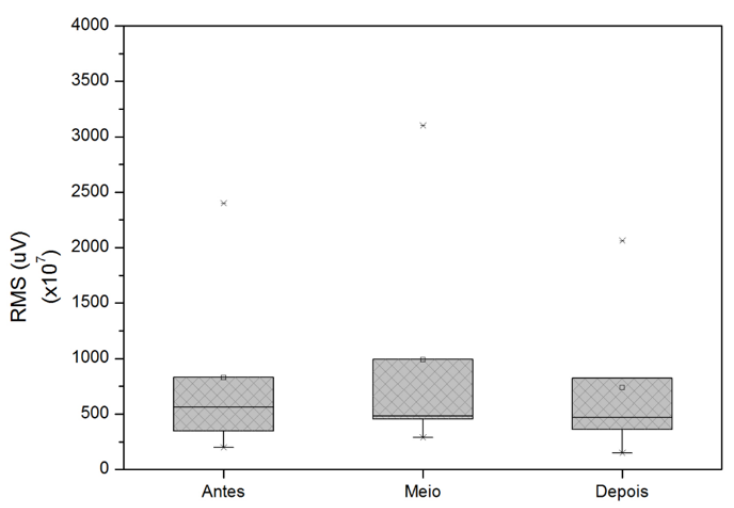

Fonte: Os autores.

Figura 6 - Box Plot da média dos valores de RMS do sinal mioelétrico do músculo tríceps braquial sadio durante contração isométrica.

EMG - Tríceps Braquial - TV

Membro Sadio

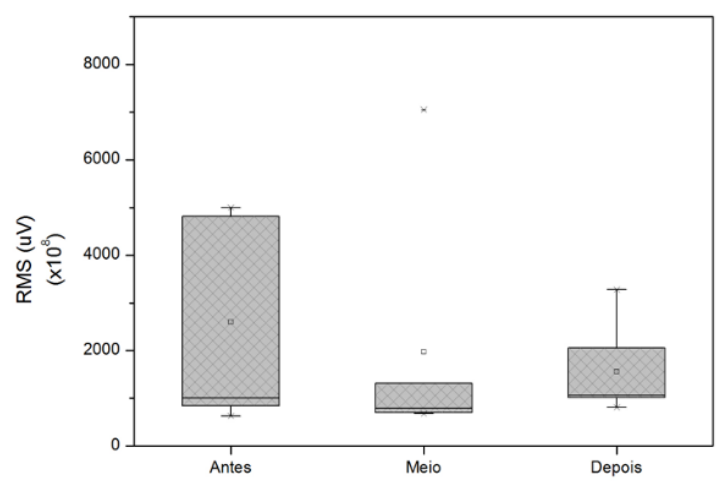

Fonte: Os autores.

Figura 7 - Box Plot da média dos valores de RMS do sinal mioelétrico do músculo deltóide médio sadio durante contração isométrica.

EMG - Deltoide Médio - TV

Membro Sadio

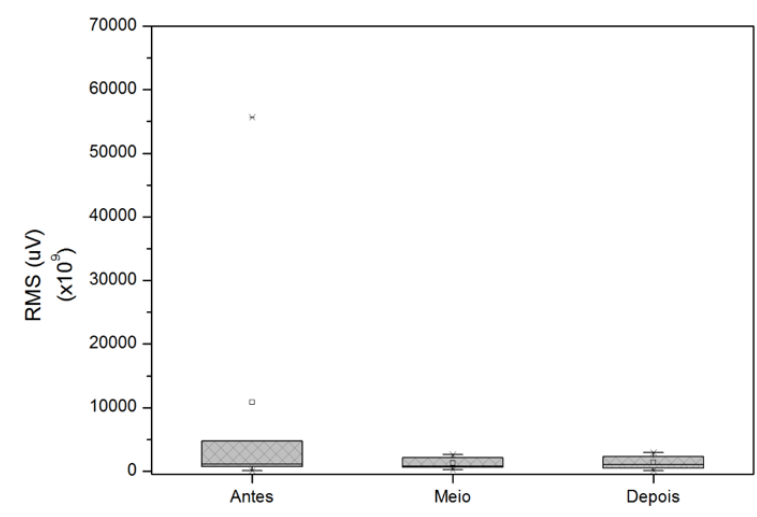

Fonte: Os autores. 


\section{DISCUSSÃO}

O desempenho da tarefa funcional exige que o indivíduo tenha capacidade para gerar e sustentar uma contração muscular. A estabilidade da força caracteriza-se por oscilações de baixa frequência na força gerada $( \pm 1 \mathrm{~Hz}$ ) e influencia o controle motor fino durante a realização da tarefa (CARLYLE; MOCHIZUKI, 2018). A disfunção gerada pelo acidente vascular encefálico impacta em déficits de força muscular, controle e coordenação do movimento voluntário, sendo o membro afetado incapaz de produzir e controlar a força (LODHA et al., 2010). A fraqueza muscular é um dos principais contribuintes para o comprometimento do membro superior, sendo atribuída à perda da ativação da via corticoespinal descendente aos motoneurônios da coluna vertebral (CHANG et al., 2013).

O presente estudo teve como objetivo avaliar os efeitos da terapia virtual no membro superior parético de indivíduos pós AVE, sendo mensuradas a força e a atividade elétrica muscular. Lee (2013) investigou os efeitos do treinamento por jogos do Xbox Kinect na força muscular, tônus muscular e nas atividades de vida diária de pacientes pós AVE. Como resultado observou aumento da força muscular no membro superior, exceto punho, e melhora da função durante as atividades de vida diária. Ikbali Afsar et al. (2018) realizaram um estudo cujo objetivo foi avaliar o efeito na terapia por realidade virtual usando o vídeo game $X$ box Kinect juntamente com a reabilitação convencional. Os autores concluíram que o treinamento com Xbox Kinect contribui para recuperação funcional do membro superior de pacientes com AVE subagudo, quando complementado à terapia convencional.

Após a intervenção através da terapia virtual realizada neste estudo, foi possível constatar resultados quantitativos na avaliação realizada por meio do dinamômetro, onde pode-se observar aumento significativo da força muscular.

A terapia por realidade virtual ganha popularidade por consistir em um sistema de fácil uso e aplicabilidade, além de aumentar a eficiência da terapia e o nível de atenção dos pacientes durante o treinamento (SCHUSTER-AMFT et al., 2018). A prática repetitiva e intensiva de atividades funcionais, que pode ser promovida pela terapia virtual, é capaz de modificar as estruturas neurais envolvidas no controle motor e no processo de aprendizagem (KIPER et al., 2018).

A eletromiografia é um recurso utilizado para avaliar o padrão da contração muscular. Um dos parâmetros fornecidos pela decomposição do sinal eletromiográfico é a amplitude, esta indica o recrutamento das unidades motoras, podendo evidenciar alterações no recrutamento de fibras musculares (DIAS, 2018). O tônus muscular aumentado presente nos portadores de AVE está associado à uma co-ativação anormal de agonista e antagonista (PUNDIK et al., 2018).

No presente estudo, o sinal EMG confirma os resultados observados pelo dinamômetro referente à força muscular, no qual foi constatado aumento significativo do sinal eletromiográfico do músculo bíceps braquial parético, seguido pela redução significativa da atividade elétrica do músculo tríceps braquial parético, sugerindo um recrutamento sinérgico de unidades motoras na relação agonista/antagonista durante a contração isométrica.

Dias (2018) realizou um estudo onde avaliou o efeito imediato da realidade virtual no membro superior parético. Foi avaliada a atividade elétrica do músculo bíceps braquial antes, durante e após a intervenção com realidade virtual. A autora não observou diferença estatística nos dados eletromiográficos do grupo controle, que teve o tratamento com fisioterapia convencional, e do grupo que participou da intervenção com realidade virtual. Contrário do que foi observado neste estudo, onde 
ouve diferença no sinal eletromiográfico após o tratamento com terapia virtual, porém após dez sessões de intervenção, não imediatamente.

Quando observados os dados eletromiográficos dos músculos bíceps e tríceps braquial sadios, não foram constatadas alterações significativas no recrutamento de fibras musculares, sugerindo que o aumento da força muscular detectado pelo dinamômetro estaria diretamente relacionado ao membro parético, descartando a possibilidade de compensação pelo membro superior sadio.

\section{CONCLUSÃO}

A terapia virtual promoveu resultados satisfatórios aos participantes com sequelas de AVE no que concerne ao aumento da força e atividade mioelétrica do membro superior parético, o que pode contribuir para o retorno do paciente às suas atividades de vida diária e melhora da qualidade de vida. Acredita-se que essa modalidade terapêutica inovadora pode ser incluída na prática clínica tanto para avaliação quanto para tratamento desses participantes.

\section{REFERÊNCIAS}

ARAMAKI, A. L. et al. Realidade virtual na reabilitação de pacientes após acidente vascular cerebral: uma revisão integrativa. Arquivos de Neuro-Psiquiatria, v. 77, n.4, p. 268-278, 2019. Doi: 10.1590/0004-282X20190025.

CARLYLE, J. K.; MOCHIZUKI, G. Influence of post-stroke spasticity on EMG-force coupling and force steadiness in biceps brachii. Journal of Electromyography and Kinesiology, v. 38, p. 48-55, 2018. Doi: 10.1016/j.jelekin.2017.11.005.

CARVALHO, R. et al. Physiotherapy based on problem-solving in upper limb function and neuroplasticity in chronic stroke patients: A case series. Journal of Evaluation in Clinical Practice, v. 24, n. 3, p. 552-560, 2018. Doi: 10.1111/jep.12921.

CHANG, S. H. et al. Spasticity, weakness, force variability, and sustained spontaneous motor unit discharges of resting spastic paretic biceps brachii muscles in chronic stroke. Muscle Nerve, v.48, n. 1, p. 85-92, 2013. Doi:

10.1002/mus.23699.

CHOI, Y. H.; PAIK, N. J. Mobile Game-based Virtual Reality Program for Upper Extremity Stroke Rehabilitation. Journal of Visualized Experiments, n. 133, p. 56241, 2018. Doi: 10.3791/56241.

DIAS, M. P. F. Efeito imediato da realidade virtual sobre a atividade eletroencefalográfica e eletromiográfica no membro superior parético após acidente vascular encefálico. 2018. 98 f. Dissertação (Mestrado em Ciências da Reabilitação) - Universidade Federal de Alfenas, Alfenas, 2018.

IKBALI AFSAR, S. et al. Virtual Reality in Upper Extremity Rehabilitation of Stroke Patients: A Randomized Controlled Trial. Journal of Stroke and Cerebrovascular Diseases, v.27, n.12, p.3473-3478, 2018. Doi:

10.1016/j.jstrokecerebrovasdis.2018.08.007. 
KHAN, F. et al. Neurorehabilitation: applied neuroplasticity. Journal of Neurology, v. 264, n. 3, p. 603-615, 2016. Doi: 10.1007/s00415-016-8307-9.

KIPER, P. et al. Virtual Reality for Upper Limb Rehabilitation in Subacute and Chronic Stroke: A Randomized Controlled Trial. Archives of Physical Medicine and

Rehabilitation, v. 99, n. 5, p. 834-842, 2018. Doi: 10.1016/j.apmr.2018.01.023.

LAUDY-EKMAN, L. Neurociências: fundamentos para reabilitação. 3ed. Rio de Janeiro: Elsevier, 2008.

LEE, G. Effects of Training Using Video Games on the Muscle Strength, Muscle Tone and Activities of Daily Living of Chronic Stroke Patients. Journal of Physical Therapy Science, v. 25, n. 5, p. 595-597, 2013. Doi: 10.1589/jpts.25.59.

LODHA, N. et al. Force control and degree of motor impairments in chronic stroke. Clinical Neurophysiology, v. 121, n. 11, p. 1952-1961, 2010. Doi: 10.1016/j.clinph.2010.04.005.

MAGGIO, M. G. et al. Virtual Reality and Cognitive Rehabilitation in People With Stroke. Journal of Neuroscience, v. 51, n. 2, p. 101-105, 2019. Doi: 10.1097/JNN.0000000000000423.

NAM, K. E. et al. When does spasticity in the upper limb develop after a first stroke? A nationwide observational study on 861 stroke patients. Journal of Clinical Neuroscience, v. 66, p. 144-148, 2019. Doi: 10.1016/j.jocn.2019.04.034.

PUNDIK, S. et al. Association of spasticity and motor dysfunction in chronic stroke. Annals of Physical and Rehabilitation Medicine, v.62, n.6, p.397-402, 2018. Doi: 10.1016/j.rehab.2018.07.006.

SCHUSTER-AMFT, C. et al. Effect of a four-week virtual reality-based training versus conventional therapy on upper limb motor function after stroke: A multicenter parallel group randomized trial. PLOS ONE, v. 13, n. 10, p. e0204455, 2018. Doi: 10.1371/journal.pone.0204455.

TANAKA, $\mathrm{H}$. et al. Spatiotemporal gait characteristic changes with gait training using the hybrid assistive limb for chronic stroke patients. Gait and Posture, v. 71, p. 205210, 2019. Doi: 10.1016/j.gaitpost.2019.05.003.

TRICCAS, L. T. et al. Predictors of upper limb spasticity after stroke? A systematic review and meta-analysis. Physiotherapy, v.105, n.2, p.163-173, 2019. Doi: 10.1016/j.physio.2019.01.004. 